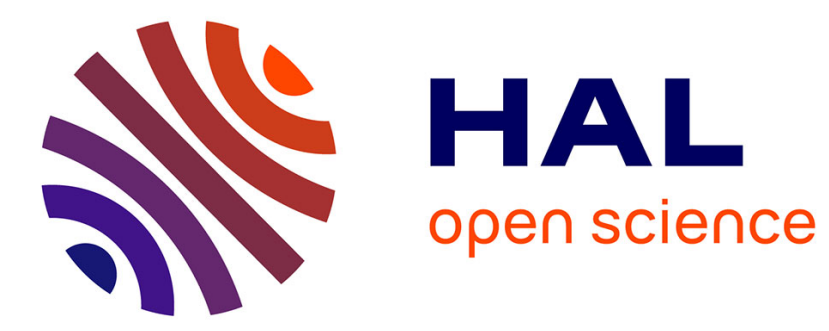

\title{
Which 3D Geometric Facial Features Give Up Your Identity?
}

\author{
Lahoucine Ballihi, Ben Amor Boulbaba, Mohamed Daoudi, Anuj Srivastava, \\ Driss Aboutajdine
}

\section{- To cite this version:}

Lahoucine Ballihi, Ben Amor Boulbaba, Mohamed Daoudi, Anuj Srivastava, Driss Aboutajdine. Which 3D Geometric Facial Features Give Up Your Identity?. International Conference on Biometrics, Mar 2012, New Delhi, India. pp.119-124, 10.1109/ICB.2012.6199768 . hal-00726082

\section{HAL Id: hal-00726082 \\ https://hal.science/hal-00726082}

Submitted on 29 Aug 2012

HAL is a multi-disciplinary open access archive for the deposit and dissemination of scientific research documents, whether they are published or not. The documents may come from teaching and research institutions in France or abroad, or from public or private research centers.
L'archive ouverte pluridisciplinaire HAL, est destinée au dépôt et à la diffusion de documents scientifiques de niveau recherche, publiés ou non, émanant des établissements d'enseignement et de recherche français ou étrangers, des laboratoires publics ou privés. 


\section{Which 3D Geometric Facial Features Give Up Your Identity ?}

\author{
Lahoucine Ballihi \\ LIFL (UMR USTL/CNRS 8022) \\ Université de Lille 1, France. \\ LRIT, Unité Associée au CNRST (URAC 29) \\ Université Mohammed V - Agdal, Maroc. \\ http://www.telecom-lille1.eu/people/ballihi/
}

\author{
Anuj Srivastava \\ Departement of Statistics \\ FSU, Tallahassee, FL 32306, USA.
}

\author{
Driss Aboutajdine \\ LRTT, Unité Associée au CNRST (URAC 29) \\ Université Mohammed V Agdal, Maroc.
}

\begin{abstract}
The $3 D$ face recognition literature has many papers that represent facial shapes as collections of curves of different kinds (level-curves, iso-level curves, radial curves, profiles, geodesic polarization, iso-depth lines, iso-stripes, etc.). In contrast with the holistic approaches, the approaches that match faces based on whole surfaces, the curve-based parametrization allows local analysis of facial shapes. This, in turn, facilitates handling of pose variations (probe image may correspond to a part of the face) or missing data (probe image is altered by occlusions. An important question is: Does the use of full set of curves leads to better performances? Among all facial curves, are there ones that are more relevant than others for the recognition task? We explicitly address these questions in this paper. We represent facial surfaces by collections of radial curves and iso-level curves, such that shapes of corresponding curves are compared using a Riemmannian framework, select the most discriminative curves (geometric features) using boosting. The experiment involving FRGCv2 dataset demonstrates the effectiveness of this feature selection by achieving $\mathbf{9 8 . 0 2 \%}$ as rank-1 recognition rate. This selection also results in a more compact signature which significantly reduces the computational cost and the storage requirements for the face recognition system.
\end{abstract}

\section{Introduction and related work}

Biometric recognition aims to use behavioral and/or physiological characteristics of people to recognize them or to verify their identities. While some biometric modalities, such as fingerprints and iris, have already reached very high level of accuracy, they have a limited use in non-cooperative scenarios. On the other hand, the less-intrusive modalities like the face and gait have not reached the desired levels of accuracy. Since face recognition is contact-less and less intrusive, it emerges as a more attractive and natural biometric for security applications. Unfortunately, image-based face recognition technologies still face difficult challenges such as lighting conditions, pose variations, occlusions, and facial expressions. In the last few years, face recognition using the $3 \mathrm{D}$ shape of face has become a major research area due to its theoretical robustness to lighting condition and pose variations. However, the problem remains due to variability in facial expressions. Several approaches have been proposed and applied to deal with deformations caused by facial expressions. In the current paper, we focus on those approaches that either use curve-based representations for faces or use some feature selection technique to optimize recognition rates:

A. Curve-based approaches. This kind of method extracts representative facial curves from $3 \mathrm{D}$ face to alter surface matching with curve matching for recognition. The key problem of these approaches is how to define schemes of curve extraction and matching able to model facial deformations? One earlier and remarkable work in proposed by Samir et al. in [19] where 3D faces were instead represented through iso-depth lines. Then, shapes of the iso-depth lines were compared, exploiting Riemannian shape analysis for planar closed curves. However, since face expressions induce strong alterations of the iso-depth lines, this approach is likely to be very sensitive to expression changes. An extension of this work using iso-geodesic curves were presented in [20].

According to Bronstein et al. study [2], the change of the geodesic distance due to facial expressions is insignifi- 
cant compared to changes of Euclidean distance. A similar study was followed in [22] by using sample points taken at the intersection between contour curves and radial profiles originated from the nose tip and calculated the euclidean distances between corresponding points of different faces. Their approach select subsets of facial curves for effective and efficient face matching. Drira et al. [8] explored the use of shapes of noses for performing partial human biometrics. They extract iso-geodesic curves then performs differential geometry-driven metrics to construct geodesics between shapes on a defined Manifold of close curves. The same framework allows statistics (means) computation for efficient search in a gallery dataset. In [7], same authors proposed similar shape analysis approach on radial curves. They models elastic deformations of facial surfaces as an optimal re-parametrization problem that they solve by Dynamic Programming. Their approach performs promising results on GAVAB database even where the probe pose is non-frontal.

Mpiperis et al. proposed an elastically deformable model algorithm [18] that establishes correspondence among a set of faces. The main limitation of this approach is the need for a large set which should also be annotated with respect to facial expressions. In [1], the authors used the geodesic distance on the face to extract iso-geodesic facial stripes. Equal-width iso-geodesic facial stripes were used as nodes of the graph and edges between nodes were labeled with descriptors, referred to as 3D Weighted Walkthroughs (3DWWs), that captured the mutual relative spatial displacement between all the pairs of points of the corresponding stripes. Face partitioning into iso-geodesic stripes and 3DWWs together provided an approximate representation of local morphology of faces that exhibits smooth variations for changes induced by facial expressions.

B. Feature selection-based approaches. Several methods have been proposed to analyze the discriminative power of different facial parts or features of the face. Daniyal et al. [6] proposed an algorithm in which the face is represented as a vector of distances between pairs of facial landmarks. They selected the landmarks by brute-forcing the possible combinations of used/unused landmarks, compared the recognition rates, and concluded that the best selection corresponded to the landmarks located around the eyes and the nose. Kakadiaris et al. [13] use an annotated face model that is deformed elastically to fit each face thus allowing the annotation of its different anatomical areas such as the nose, eyes, mouth, etc. To handle with expressions, the authors classify faces using wavelet coefficients representing face areas that remain unaffected by facial expressions, such as eyes and nose. However, the best recognition rate is achieved when the whole face is used, which implies that rejection of deformable parts of the face leads to the loss of valuable discriminative information. In [4], a multi-nose regions matching was proposed. Primary results show substantial improvement over matching the shape of a single larger frontal face region. Faltemier et al. were extended this method in [9] by considering a set of 38 regions that densely cover the face and selecting the best-performing subset of 28 regions to perform matching using the ICP algorithm. Cadavid et al. [3] present a novel method for 3D face recognition using adaboosted geodesic distance features. They use a generic model and compute the geodesic distance between anatomical point pairs across each conformed generic model.

More recently, Wang et al. [23] compute a signed shape difference map (SSDM) between two aligned 3D faces as a intermediate representation for the shape comparison. Based on the SSDMs, three kinds of features were used to encode both the local similarity and the change characteristics between facial shapes. The most discriminative local features were selected optimally by boosting and trained as weak classifiers for assembling three collective strong classifiers. The individual features were of the type : Haarlike, Gabor, and Local Binary Pattern (LBP). Using similar features, the authors in [16] proposed to design a feature pooling and ranking scheme in order to collect various types of low-level geometric features, such as curvatures, and rank them according to their sensitivities to facial expressions. They applied sparse representations to the collected low-level features and achieved good results on GAVAB database.

In this category of approaches, based on curves, facial shape comparison return to shape comparison of their curves. But, does the use of all the curves on the face lead to better performances?, and among the facial curves is there any ones more relevant than others?

\section{Geometric shape analysis of facial curves}

We start by considering a facial curve $\beta$ in $\mathbb{R}^{3}$. It is natural to parametrize it using $\beta: \mathbb{S}^{1} \rightarrow \mathbb{R}^{3}$. Note that the parameterization is not assumed to be arc-length; we allow a larger class of parameterizations for improved analysis. To analyze the shape of $\beta$, we shall represent it mathematically using a square-root velocity function (SRVF), proposed in $[12,21]$, and denoted by $q(t)$, according to:

$$
q(t) \doteq \frac{\dot{\beta}(t)}{\sqrt{\|\dot{\beta}(t)\|}}
$$

Where $\|$.$\| is the Euclidean norm and q(t)$ is a special function that captures the shape of $\beta$ and is particularly convenient for shape analysis, as we describe next. The conventional metric for comparing the elastic shape of the curves becomes an $\mathbb{L}^{2}$ metric under the representation [12, 21]. Depends on the topology of the curve (close or open), we recall following shape analysis foundations of facial curves. 


\subsection{Radial open curves}

We define the set of open curves in $\mathbb{R}^{3}$ by $\mathcal{C}=\{q$ : $\left.I \rightarrow \mathbb{R}^{3} \mid\|q\|=1\right\} \subset \mathbb{L}^{2}\left(I, \mathbb{R}^{3}\right)$, with the $\mathbb{L}^{2}$ metric on its tangent spaces, $\mathcal{C}$ becomes a Riemannian manifold. In particular, since the elements of $\mathcal{C}$ have a unit $\mathbb{L}^{2}$ norm, $\mathcal{C}$ is a hypersphere in the Hilbert space $\mathbb{L}^{2}\left(I, \mathbb{R}^{3}\right)$. In order to compare the shapes of two radial curves, we can compute the distance between them in $\mathcal{C}$ under the chosen metric. This distance is defined to be the length of the (shortest) geodesic connecting the two points in $\mathcal{C}$. Since $\mathcal{C}$ is a sphere, the formulas for the geodesic and the geodesic length are already well known. The geodesic length between any two points $q_{1}, q_{2} \in \mathcal{C}$ is given by:

$$
d_{c}\left(q_{1}, q_{2}\right)=\cos ^{-1}\left(\left\langle q_{1}, q_{2}\right\rangle\right),
$$

and the geodesic path $\alpha:[0,1] \rightarrow \mathcal{C}$, is given by:

$$
\alpha(\tau)=\frac{1}{\sin (\theta)}\left(\sin ((1-\tau) \theta) q_{1}+\sin (\theta \tau) q_{2}\right),
$$

where $\theta=d_{c}\left(q_{1}, q_{2}\right)$.

It is easy to see that several elements of $\mathcal{C}$ can represent curves with the same shape. For example, if we rotate a face in $\mathbb{R}^{3}$, and thus its facial curves, we get different SRVFs for the curves but their shapes remain unchanged. Another similar situation arises when a curve is re-parametrized; a re-parameterization changes the SRVF of curve but not its shape. In order to handle this variability, we define orbits of the rotation group $S O(3)$ and the re-parameterization group $\Gamma$ as equivalence classes in $\mathcal{C}$. Here, $\Gamma$ is the set of all orientation-preserving diffeomorphisms of $I$ (to itself) and the elements of $\Gamma$ are viewed as re-parameterization functions. For example, for a curve $\beta: I \rightarrow \mathbb{R}^{3}$ and a function $\gamma \in \Gamma$, the curve $\beta_{\alpha} \circ \gamma$ is a reparameterization of $\beta_{\alpha}$. The corresponding SRVF changes according to $q(t) \mapsto \sqrt{\dot{\gamma}(t)} q(\gamma(t))$. We define the equivalent class containing $q$ as:

$$
[q]=\{\sqrt{\dot{\gamma}(t)} O q(\gamma(t)) \mid O \in S O(3), \quad \gamma \in \Gamma\},
$$

The set of such equivalence class is called the shape space $\mathcal{S}$ of elastic curves [12]. To obtain geodesics and geodesic distances between elements of $\mathcal{S}$, one needs to solve the optimization problem. The resulting shape space is the set of such equivalence classes:

$$
\mathcal{S} \doteq \mathcal{C} /(S O(3) \times \Gamma)
$$

We denote by $d_{s}\left(\beta_{\alpha_{1}}, \beta_{\alpha_{2}}\right)$ the geodesic distance between the corresponding equivalence classes $\left[q_{1}\right]$ and $\left[q_{2}\right]$ in $\mathcal{S}$. The figure 1 illustrates some examples of geodesic paths between facial surfaces and associated collections of radial curves.

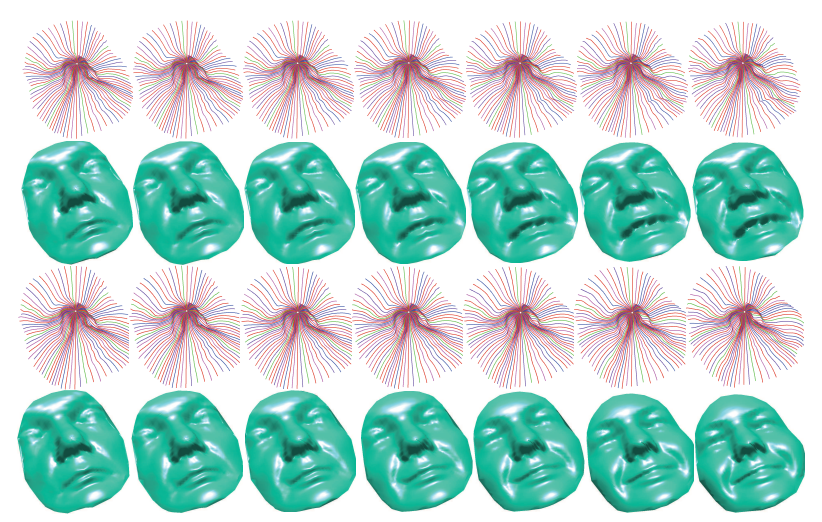

Figure 1: Examples of intra- and inter-class geodesics between facial surfaces and their associated radial curves. The first two rows give an intra-class (same person, different expressions) geodesic path, whereas the remaining two rows show an inter-class (different subjects) geodesic path.

\subsection{Iso-level closed curves}

In the same manner, we define the set of closed curves in $\mathbb{R}^{3}$ by $\tilde{\mathcal{C}}=\left\{q: \mathbb{S}^{1} \rightarrow \mathbb{R}^{3} \mid \int_{\mathbb{S}_{1}} q(t)\|q(t)\| d t=\right.$ 0\} $\left.\subset \mathbb{L}^{2}\left(\mathbb{S}^{1}, \mathbb{R}^{3}\right)\right\}$, where $\mathbb{L}^{2}\left(\mathbb{S}^{1}, \mathbb{R}^{3}\right)$ denotes the set of all integrable functions from $\mathbb{S}^{1}$ to $\mathbb{R}^{3}$. The quantity $\int_{\mathbb{S}^{1}} q(t)\|q(t)\| d t$ is the total displacement in $\mathbb{R}^{3}$ while moving from the origin of the curve until the end. When it is zero, the curve is closed. Thus, the set $\tilde{\mathcal{C}}$ represents the set of all closed curves in $\mathbb{R}^{3}$. It is called a pre-shape space since curves with same shapes but different orientations and reparameterizations can be represented by different elements of $\tilde{\mathcal{C}}$. To define a shape, its representation should be independent of its rotations and reparameterization. This is obtained mathematically by a removing the rotation group $S O(3)$ and the re-parameterization group $\Gamma$ from $\tilde{\mathcal{C}}$. As described in $[12,21]$, we define the orbits of the rotation group $S O(3)$ and the re-parameterization group $\Gamma$ as equivalence classes in $\tilde{\mathcal{C}}$. The resulting shape space is :

$$
\tilde{\mathcal{S}} \doteq \tilde{\mathcal{C}} /(S O(3) \times \Gamma)
$$

To define geodesics on pre-shape and shape spaces we need a Riemannian metric. For this purpose we inherit the standard $\mathbb{L}^{2}$ metric the large space $\mathbb{L}^{2}\left(\mathbb{S}^{1}, \mathbb{R}^{3}\right)$. For any $u, v \in$ $\mathbb{L}^{2}\left(\mathbb{S}^{1}, \mathbb{R}^{3}\right)$, the standard $\mathbb{L}^{2}$ inner-product is given by:

$$
\langle\langle u, v\rangle\rangle=\int_{\mathbb{S}^{1}}\langle u(t), v(t)\rangle d t .
$$

The computation of geodesics and geodesic distances utilize the intrinsic geometries of these spaces. While the detailed description of the geometries of $\tilde{\mathcal{C}}$ and $\tilde{\mathcal{S}}$ are given in $[12,21]$, we briefly mentioned the tangent and normal spaces of $\tilde{\mathcal{C}}$. Now, an important tool in our framework is the 
construction of a geodesic path between two elements of $\tilde{\mathcal{S}}$, under the Riemannian metric given by equation 5. Given two curves $\beta_{\lambda_{1}}$ and $\beta_{\lambda_{2}}$, represented by their SRVF respectively $q_{1}$ and $q_{2}$, we need to find a geodesic path between the orbits $\left[q_{1}\right]$ and $\left[q_{2}\right]$ in the space $\tilde{\mathcal{S}}$. We use in this context, a numerical method called the path-straightening method [15] which connects the two points $\left[q_{1}\right]$ and $\left[q_{2}\right]$ an arbitrary path $\alpha$ and then updates this path repeatedly in the negative direction of the gradient of energy given by:

$$
E[\alpha]=\frac{1}{2} \int_{s}\langle\langle\dot{\alpha}(s), \dot{\alpha}(s)\rangle\rangle d s
$$

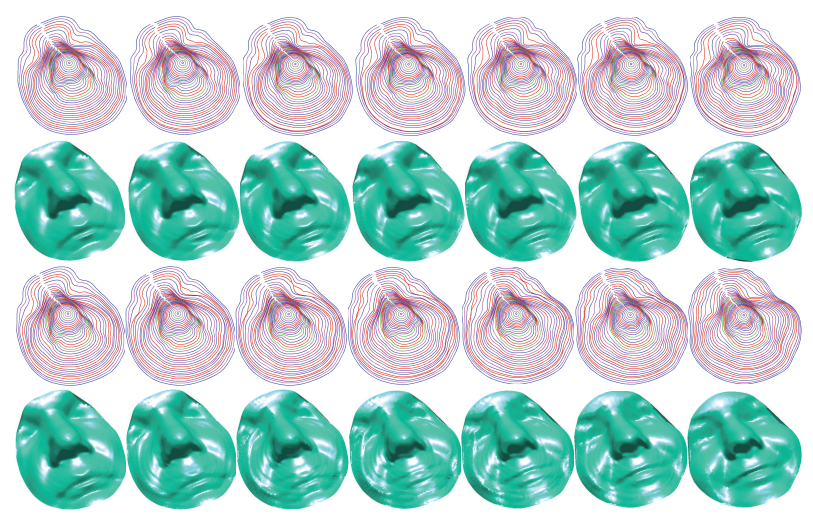

Figure 2: Examples of intra- and inter-class geodesics between facial surfaces and their associated iso-level curves. The first two rows give an intra-class (same person, different expressions) geodesic path, whereas the remaining two rows show an inter-class (different subjects) geodesic path.

It has been proven in [15] that the critical points of $E$ are geodesic paths in $\tilde{\mathcal{S}}$. We denote by $d_{\tilde{s}}\left(\beta_{\lambda_{1}}, \beta_{\lambda_{2}}\right)$ the geodesic distance between the corresponding equivalence classes $\left[q_{1}\right]$ and $\left[q_{2}\right]$ in $\tilde{\mathcal{S}}$. The figure 2 illustrates some examples of geodesic paths between facial surfaces and associated collections of iso-level curves.

\section{Boosting for geometric feature selection}

Radial and iso-level curves capture locally the shape of the faces. However, their comparison under different expressions runs into trouble. In fact, their shapes are affected by changes in facial expressions. For that purposes, we introduce a feature selection step to identify (or localize) the most stable and most discriminative curves. We propose to use the well-known machine learning algorithm, AdaBoost, introduced by Freund and Schapire in [10]. Boosting is based on iterative selection of weak classifiers by using a distribution of training samples. At each iteration, the best (relevant) weak classifier is provided and weighted by the quality of its classification. In practice, the individual iso- level curves and radial curves are used as the weak classifiers. After $M$ iterations, the most relevant $T(T<M)$ facial curves are returned by the algorithm.

To train and test the boosting algorithm, we use the FRGCv2 dataset which contains more than 4000 scans of 466 subjects. For each curve (radial or iso-level), we compute the All vs. All similarity matrices. We then discard the parts of these matrices corresponding to comparisons of neutral scan versus remaining comparisons to be used for the On-line testing phase. Remaining parts are used to train the boosting algorithm. Thus, we disjoint the training and the testing sets.In these areas of the matrices, we extract two kind of scores $(i)$ the match scores (intra-personal comparisons) and (ii) the non-match scores (inter-personal comparison). Both scores lists represent the input of the boosting algorithm. More formally, we consider a set of pairs $\left(x_{n}^{\alpha, \lambda}, y_{n}\right)_{1<n<N}$ where $x_{n}^{\alpha, \lambda}$ is a similarity score between two curves at the same level $\alpha, \lambda$ and $y_{n}$ can take two values: 0 in the case of non-match score and 1 in the case of match score. For each curve $\beta_{j}$, the weak learner determines the optimal threshold classification function, such that the minimum number of samples are misclassified. A weak classifier $h_{j}\left(x_{n}^{k}\right)$ thus consists of a geometric feature $\beta_{j}$ and a threshold $\theta$, such that

$$
h_{j}\left(x_{n}^{k}\right)= \begin{cases}1 & \text { if } x_{n}^{k}<\theta(\text { intra-personal) } \\ 0 & \text { otherwise. (inter-personal) }\end{cases}
$$

$h_{t}$ denotes for the weak hypothesis given by $h_{t}: \mathcal{X} \rightarrow$ $\{0,1\}$. The output of Boosting algorithm is a set of $T$ weak classifiers based on selected iso-level or radial curves. The set of selected curves is given in figure 3. The first row of this figure shows location of selected curves on different sessions of the some person with different expressions whereas, the second row gives curves location on different subjects. We note that the boosting algorithm selects isocurves on the nasal region, which is stable under expressions and radial curves avoiding two parts. The first one is the lower part of the face since its shape is affected by expressions, particularly when the mouth is open. The second area corresponds to the eye/eyebrow regions. Shapes of radial curves passing throw this regions changes when convey expressions. In contrast, the most stable area cover the nasal/forehead regions.

To show performances improvement of the curve selection step, different graphs in figure 4 plot the rate of False Acceptance versus the rate of False Rejection (ROC/DET curves) for different configurations. Minimum errors are given by fusing scores of selected radial and selected isolevel curves (black curve in figure 4). We note also that the selection performed on radial curves only or iso-level curves only minimizes the errors compared to the use of all radial curves or iso-level curves, respectively. 


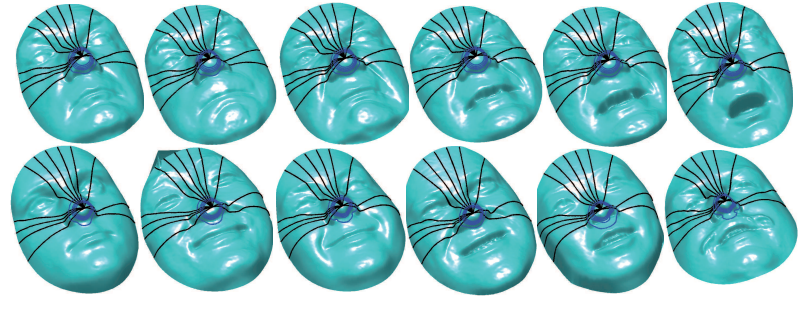

Figure 3: The most discriminative radial and iso-level curves selected by Boosting, given on different faces.

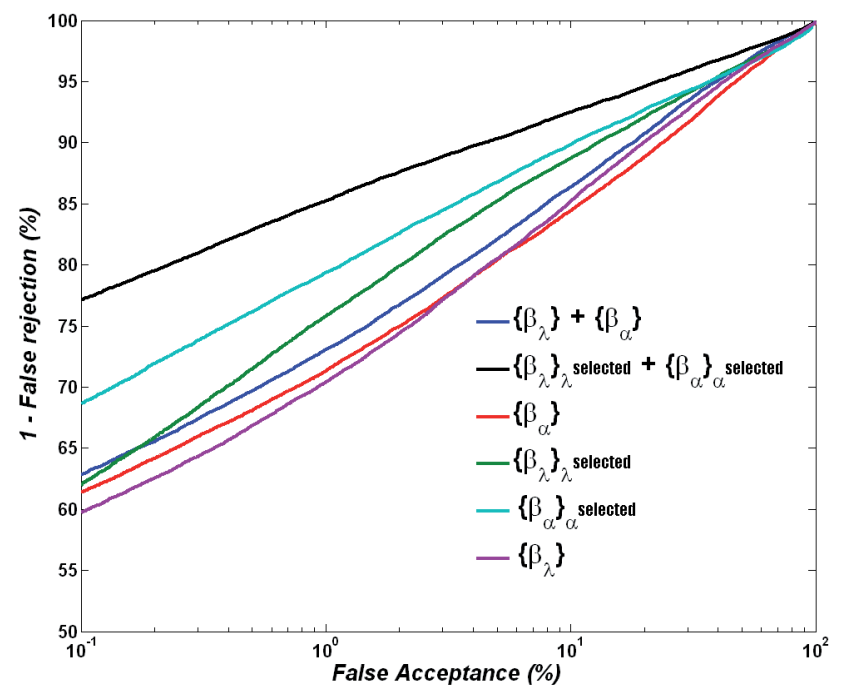

Figure 4: ROC curves produced from the training set for (a) All curves radial ans iso-level curves (b) Selected curves (c) selected radial (d) selected iso-level (e) radial only (f) isolevel only.

Once, the training step ended and most stable and discriminative curves selected, we can move to the on-line testing step. It consists on comparing faces $s^{1}$ and $s^{2}$ by the fusion of scores related to selected curves as following:

$d\left(s^{1}, s^{2}\right)=\int_{\lambda_{\text {selected }}} d_{s}\left(\beta_{\alpha}^{1}, \beta_{\alpha}^{2}\right) d \lambda+\int_{\alpha_{\text {selected }}} d_{\tilde{s}}\left(\beta_{\lambda}^{1}, \beta_{\lambda}^{2}\right) d \alpha$.

\section{Experimental results}

We conducted identification experiments following the FRGCv2 protocol which consist to keep in the Gallery the earliest scans 466 and consider remaining scans as test images (Probe). Our approach achieved $98.02 \%$ as rank-1 recognition rate which is competitive compared to stateof-the-art performances. The table 1 gives identifications results of previous approaches. Using a combination of iso- curves and radial curves, selected using Machine Learning technique on training dataset, the proposed algorithm outperforms most of related work. Wang et al. [23] achieved the best result $98.3 \%$ which means ten more recognized probes in comparison to our approach. We recall that their method uses boosting technique to select among other king of descriptors (Haar-like, Gabor, and Local Binary Pattern (LBP)) computed on the Shape Difference Map between faces.

Another interesting result of the present approach is reported in Table 2. This table shows performances and time performances of different configurations. The proposed approach achieved the best performances using compact signature which has direct impact in the computational efficiency of the face recognition system in term of storage requirement and computational time $(0.68(\mathrm{~s})$ instead of $1.6(\mathrm{~s}))$. We also find that the radial curves give better performance compared to the iso-level curves. The number of radial curves, that pass through the region of the mouth, the region where the curves change seriously it shapes, is smaller than the total number of radial curves. However, the most of the iso-level curves passes through this region(open mouth), this is why all selected iso-level curves are from the nasal region, this confirms the robustness of this region to facial expressions.

\section{Conclusions and future directions}

In this paper we demonstrate the effectiveness of facial curves selection before performing faces analysis and comparison. The main ingredient is the use of Machine Learning technique (Boosting) to select relevant features under expressions. First, we consider both radial and iso-level curves which are significantly used in the literature. Then, most discriminative curves (features) are selected optimally by boosting and trained as weak classifiers for building a strong classifier. The experiments, carried out on FRGCv2 including neutral and non-neutral images, demonstrates the effectiveness of the proposed study. With 17 curves 12 radial and 5 iso-level, a rank-1 recognition rate of $\mathbf{9 8 . 0 2 \%}$ was achieved, the algorithm execution time was on the order of $\mathbf{0 . 6 8}$ seconde to compare two faces with selected curves instead of $\mathbf{2 . 6 4}$ seconde with all curves. The boosting selects those curves passing through stable regions on the face. As future direction, this approach could be used for facial curves selection in expression recognition and extended to include in addition to boosting other Machine Learning techniques.

\section{Acknowledgements}

This research is supported in part by the ANR under the projects ANR FAR 3D ANR-07-SESU-004 and 3D Face Analyzer ANR 2010 INTB 030102. 
Table 1: Comparison with state-of-the-art approaches on FRGCv2 (rank-1 recognition rate).

\begin{tabular}{|c|c|c|c|c|c|c|c|c|c|}
\hline \multirow[b]{2}{*}{ Methods } & \multicolumn{3}{|c|}{ A. Curve-based representation } & \multicolumn{4}{|c|}{ B. Feature selection-based } & \multicolumn{2}{|c|}{ Others } \\
\hline & $\begin{array}{c}\text { ter Haar } \\
{[22]}\end{array}$ & $\begin{array}{c}\text { Berretti }^{(*)} \\
{[1]}\end{array}$ & $\begin{array}{c}\text { Mpiperis } \\
{[18]}\end{array}$ & $\begin{array}{c}\text { Faltemier } \\
{[9]}\end{array}$ & $\begin{array}{c}\text { Kakadiaris } \\
{[14]}\end{array}$ & $\begin{array}{c}\text { Wang } \\
{[23]}\end{array}$ & $\begin{array}{c}\text { Hang } \\
{[11]}\end{array}$ & $\begin{array}{c}\operatorname{Mian}^{(*)} \\
{[17]}\end{array}$ & $\begin{array}{c}\text { Cook } \\
{[5]}\end{array}$ \\
\hline Rank-1 & $97 \%$ & $94.1 \%$ & - & $97.2 \%$ & $97 \%$ & $98.3 \%$ & $97.2 \%$ & $91.9 \%$ & $92.9 \%$ \\
\hline Our & & & & & $2 \%$ & & & & \\
\hline
\end{tabular}

(*) "E-N-S" means the earliest neutral scan in the gallery and the remaining as probes.

Table 2: Rank-1/Computation Cost (in sec) for different configurations.

\begin{tabular}{||c||c||c|}
\hline Rank-1/Time(s) & All & Selected \\
\hline Radial & $88.65 \% / 1.6$ & $89.04 \% / 0.48$ \\
\hline Iso-level & $66.51 \% / 1.04$ & $85.65 \% / 0.20$ \\
\hline Fusion & $91.81 \% / 2.64$ & $\mathbf{9 8 . 0 2 \% / 0 . 6 8}$ \\
\hline
\end{tabular}

\section{References}

[1] S. Berretti, A. D. Bimbo, and P. Pala. 3d face recognition using isogeodesic stripes. IEEE Trans. Pattern Anal. Mach. Intell., 32(12):2162-2177, 2010.

[2] A. M. Bronstein, M. M. Bronstein, and R. Kimmel. Expression-invariant representations of faces. IEEE Transactions on Image Processing, 16(1):188-197, 2007.

[3] S. Cadavid, J. Zhou, and M. Abdel-Mottaleb. Determining discriminative anatomical point pairings using adaboost for 3d face recognition. In ICIP, pages 49-52, 2009.

[4] K. I. Chang, K. W. Bowyer, and P. J. Flynn. Multiple nose region matching for $3 \mathrm{D}$ face recognition under varying facial expression. IEEE Trans. Pattern Anal. Mach. Intell., 28(10):1695-1700, 2006.

[5] J. A. Cook, V. Chandran, and C. B. Fookes. 3d face recognition using log-gabor templates. In British Machine Vision Conference, Edinborugh, Scotland, 2006.

[6] F. Daniyal, P. Nair, and A. Cavallaro. Compact signatures for $3 \mathrm{~d}$ face recognition under varying expressions. In $A d$ vanced Video and Signal Based Surveillance, 2009. AVSS '09. Sixth IEEE International Conference on, pages $302-$ 307, sept. 2009.

[7] H. Drira, B. B. Amor, M. Daoudi, and A. Srivastava. Pose and expression-invariant $3 \mathrm{~d}$ face recognition using elastic radial curves. In $B M V C$, pages 1-11, 2010.

[8] H. Drira, B. B. Amor, A. Srivastava, and M. Daoudi. A riemannian analysis of 3D nose shapes for partial human biometrics. In IEEE International Conference on Computer Vision, pages 2050-2057, 2009.

[9] T. Faltemier, K. Bowyer, and P. Flynn. A region ensemble for 3-d face recognition. IEEE Trans. on Information Forensics and Security, 3(1):62-73, 2008.

[10] Y. Freund and R. E. Schapire. A decision-theoretic generalization of on-line learning and an application to boosting. In EuroCOLT '95: Proceedings of the Second European Conference on Computational Learning Theory, pages 23-37, London, UK, 1995. Springer-Verlag.
[11] D. Huang, M. Ardabilian, Y. Wang, and L. Chen. A novel geometric facial representation based on multi-scale extended local binary patterns. In Automatic Face Gesture Recognition and Workshops (FG 2011), 2011 IEEE International Conference on, pages $1-7$, march 2011.

[12] S. H. Joshi, E. Klassen, A. Srivastava, and I. H. Jermyn. A novel representation for efficient computation of geodesics between $n$-dimensional curves. In IEEE CVPR, pages 1-7, 2007.

[13] I. Kakadiaris, G. Passalis, G. Toderici, M. Murtuza, Y. Lu, N. Karampatziakis, and T. Theoharis. Three-dimensional face recognition in the presence of facial expressions: An annotated deformable model approach. IEEE Trans. Pattern Anal. Mach. Intell., 29(4):640 -649, april 2007.

[14] I. A. Kakadiaris, G. Passalis, G. Toderici, M. N. Murtuza, Y. Lu, N. Karampatziakis, and T. Theoharis. Threedimensional face recognition in the presence of facial expressions: An annotated deformable model approach. IEEE Trans. Pattern Anal. Mach. Intell., 29(4):640-649, 2007.

[15] E. Klassen and A. Srivastava. Geodesics between 3D closed curves using path-straightening. In Proceedings of ECCV, Lecture Notes in Computer Science, pages 95-106, 2006.

[16] X. Li, T. Jia, and H. Z. 0002. Expression-insensitive 3d face recognition using sparse representation. In $C V P R$, pages 2575-2582, 2009.

[17] A. Mian, M. Bennamoun, and R. Owens. An efficient multimodal 2d-3d hybrid approach to automatic face recognition. IEEE Trans. Pattern Anal. Mach. Intell., 29(11):1927 -1943, nov. 2007.

[18] I. Mpiperis, S. Malassiotis, and M. G. Strintzis. 3-d face recognition with the geodesic polar representation. IEEE Trans. on Information Forensics and Security, 2(3-2):537547, 2007.

[19] C. Samir, A. Srivastava, and M. Daoudi. Three-dimensional face recognition using shapes of facial curves. IEEE Trans. Pattern Anal. Mach. Intell., 28(11):1847-1857, 2006.

[20] C. Samir, A. Srivastava, M. Daoudi, and E. Klassen. An intrinsic framework for analysis of facial surfaces. International Journal of Computer Vision, 82(1):80-95, 2009.

[21] A. Srivastava, E. Klassen, S. H. Joshi, and I. H. Jermyn. Shape analysis of elastic curves in euclidean spaces. IEEE Trans. Pattern Anal. Mach. Intell., 33(7):1415-1428, 2011.

[22] F. B. ter Haar and R. C. Veltkamp. A 3d face matching framework for facial curves. Graph. Models, 71(2):77-91, 2009.

[23] Y. Wang, J. Liu, and X. Tang. Robust 3d face recognition by local shape difference boosting. IEEE Trans. Pattern Anal. Mach. Intell., 32:1858-1870, October 2010. 